\title{
A cadeia de valor em saúde: tornar os sistemas de saúde mais sustentáveis
}

Casimiro Cavaco Dias*

\section{RESUMO}

Face à actual crise económica, os sistemas de saúde em Portugal e na Europa têm-se centrado em obter ganhos de eficiência de serviços. Contudo, as abordagens de avaliação de custos constituem uma barreira à criação de valor em saúde e originam medidas de controlo de custos que podem ser inefectivas.

Controlar custos sem analisar os resultados em saúde é contra produtivo, dado conduzir a eventuais poupanças a curto prazo que limitam a sua efectividade e comprometem a sustentabilidade a longo prazo. A evidência aponta que os investimentos nos cuidados de saúde primários e prevenção da doença podem reduzir a pressão crescente sobre os serviços de urgência, muito mais dispendiosos.

O valor, enquanto resultado em saúde combinado com os custos, deve definir a estrutura de melhoria de desempenho no sistema de saúde. O foco no valor em saúde permite libertar o potencial de inovação existente na sociedade Portuguesa. Esta sociedade mais inovadora será necessariamente mais saudável, mais produtiva e mais resiliente a futuras crises financeiras.

Palavras-chave: Inovação; Tecnologias médicas; Sustentabilidade; Portugal; Serviço Nacional de Saúde.

\section{INTRODUÇÃO}

A actual crise económica traduz-se em importantes consequências ao nível do desempenho do sistema da saúde. Com o aumento da procura de cuidados de saúde, assiste-se ao aumento da pressão financeira. Por último, a crise afeta sobretudo os grupos mais vulneráveis da população, nomeadamente o número crescente de desempregados. $^{1-2}$

Na resposta à actual crise, os países europeus recorreram a uma combinação de instrumentos políticos. Na sua maioria, a crise foi utilizada para promover ganhos de eficiência, sobretudo nos sectores hospitalar e farmacêutico. Contudo, verifica-se um défice de iniciativas para criar valor através da promoção da saúde e da prevenção da doença. Tendo em conta o crescimento da despesa em saúde, o foco em melhorias de eficiência dos serviços não permite, só por si, assegurar a sustentabilidade do sistema de saúde., ${ }^{1,3-4}$

*Health Economist. Health Systems Strengthening Specialist. World Health Organization
Torna-se necessário promover a inovação em saúde, para além do foco das novas tecnologias médicas em diagnóstico e tratamento. É essencial aproveitar as oportunidades em áreas subdesenvolvidas, como os comportamentos saudáveis e de utilização racional de serviços de saúde, assim como criar novos modelos de serviços de saúde centrados na pessoa e mais próximos da comunidade. Contudo, na verdade, os sistemas de saúde mantêm o seu modelo hospitalocêntrico, onde cerca de $97 \%$ do orçamento da saúde dos países europeus é destinado ao diagnóstico e tratamento de doenças, enquanto apenas 3\% é investido em prevenção. ${ }^{5}$

Por outro lado, apesar de importantes mudanças transformadoras em serviços de saúde, há ainda evidência limitada sobre o impacto da inovação na melhoria da saúde da população e melhoria do desempenho dos sistemas de saúde.

\section{O IMPACTO DA INOVAÇÃO EM SAÚDE}

Hoje, a análise do sector da saúde em Portugal sugere que a relação entre a inovação e o desempenho é 
marcada por uma visão demasiado estrita ao nível do desempenho dos serviços de saúde. Contudo, o impacto da inovação vai para além do desempenho organizacional, com potencial para transformar o sistema de saúde e acrescentar valor para todos os portugueses. ${ }^{6}$

Aqui, o valor é definido como os resultados em saúde atingidos por cada euro investido. Este valor é o que realmente interessa às pessoas, reunindo os objectivos partilhados por todos os principais actores do sistema de saúde, assegurando assim a sua sustentabilidade.

Promover a inovação no sistema de saúde requer a medição do seu valor através da análise simultânea dos resultados em saúde e dos seus custos associados. Por exemplo, estudos anteriores demonstram que o retorno de inovações específicas corresponde a ganhos em saúde e a redução de custos de tratamento no valor máximo de 38 dólares por cada dólar investido. ${ }^{4}$ Estes investimentos, além de melhorarem a saúde e a qualidade de vida das pessoas, resultam ainda em importantes benefícios económicos. Estimativas indicam que o aumento da esperança de vida acrescentou 3,2 mil milhões de dólares por ano à economia dos Estados Unidos da América.

Por outro lado, nenhum serviço de saúde, só por si, e de forma isolada, é capaz de criar inovação com valor. Pelo contrário, a inovação acontece sobretudo em redes de cooperação que se traduzem no ciclo de cuidados de saúde. Ao avaliar os resultados e custos ao longo do ciclo de cuidados de saúde torna-se possível garantir a realocação de despesas em serviços mais eficientes, evitar a duplicação de serviços sem valor acrescentado, assim como promover serviços mais efectivos e próximos dos cidadãos. ${ }^{7-9} \mathrm{O}$ valor para a pessoa é criado pelos esforços combinados ao longo de uma cadeia de cuidados de saúde. Os benefícios de uma intervenção específica dependerão da efectividade de outras intervenções ao longo desta cadeia. Por conseguinte, o compromisso para acrescentar valor deverá ser partilhado entre as organizações envolvidas. ${ }^{10}$

Esta visão holística do ciclo de cuidados de saúde foca a atenção em efeitos que são invisíveis em análises verticais. $O$ valor acrescentado de uma determinada intervenção específica pode ser reduzido ou até contraprodutivo em outras fases do ciclo de cuidados. A evidência aponta para que investimentos na prevenção e gestão da doença tenham potencial de melhorar o seu valor global, reduzindo os custos e/ou melhorando a efectividade.

Ao invés da mera soma de intervenções ou serviços específicos, o valor em saúde representa o resultado do ciclo de cuidados. As decisões de investimento devem ser analisadas em termos do seu valor ao longo da cadeia de valor e não apenas através da análise de custoefectividade de intervenções isoladas, que são muitas vezes consideradas fora do seu contexto epidemiológico e social. ${ }^{11-12}$

\section{CONCLUSÃO}

Como será o sistema de saúde quando todos os actores se focarem no valor em saúde? Os serviços serão organizados em torno da pessoa e coordenados ao longo do ciclo de cuidados. Ao colocar a pessoa no centro dos serviços de saúde, promove-se a autogestão da saúde, assim como a prestação de serviços de saúde custo-efectivos na comunidade e mais próximos das pessoas. A redefinição do sistema de saúde passa por reforçar os cuidados de saúde primários com o centro de ligação a todos os outros níveis de cuidados. Os centros de saúde dispõem de mais informação e opções para os seus utilizadores, assim como de programas integrados de prevenção e gestão da doença. Desta forma, assegura-se a continuidade dos cuidados através da coordenação entre os serviços ao longo de uma cadeia de valor. Por último, esta transformação passa ainda por promover uma cultura de melhoria contínua da qualidade com base na melhor evidência disponível.

\section{REFERÊNCIAS BIBLIOGRÁFICAS}

1. Mladovsky P, Srivastava D, Cylus J, Karanikolos M, Evetovits T, Thomson $\mathrm{S}$, et al. Health policy responses to the financial crisis in Europe. Copenhagen:World Health Organization; 2012.

2. Morgan D, Astolfi R. Health spending growth at zero: which countries, which sectors are most affected? Paris: OECD; 2013.

3. Cutler DM, Rosen AB, Vijan S. The value of medical spending in the United States, 1960-2000. N Engl J Med. 2006;355(9):920-7.

4. Luce BR, Mauskopf J, Sloan FA, Ostermann J, Paramore LC. The return on investment in health care: from 1980 to 2000. Value Health. 2006;9(3):146-56.

5. World Health Organization. Review of public health services and capacities in Europe. Copenhagen:World Health Organization; 2012.

6. Cavaco-Dias C. O valor da inovação: criar o futuro do sistema de saúde. Coimbra: Almedina; 2015. ISBN 9789724061665

7. Robert Wood Johnson Foundation. What are the biggest drivers of cost in U.S. Health Care? [Internet]. Princeton: RWJF; 2011. Available from: 
http://www.rwjf.org/en/library/research/2011/07/what-are-thebiggest-drivers-of-cost-in-u-s--health-care-.html

8. Mulgan G, Albury D. Innovations in the public sector [Internet]. London: Cabinet Office; 2003. Available from:www.alnap.org/pool/files/innovation-in-the-public-sector.pdf

9. Murphy KM, Topel RH. The value of health and longevity [Internet]. Cambridge: National Bureau of Economic Research; 2005. Available from: http://www.nber.org/papers/w11405

10. Kelly G, Mulgan G, Muers S. Creating public value: an analytical framework for public service reform. London: Strategy Unit, Cabinet Office: 2002.

11. Porter $M$, Millar V. How information gives you competitive advantage. Harv Bus Rev. 1985;63(4):149-60.
12. Porter ME. What is value in health care? N Eng J Med. 2010;363 (26):2477-81.

\section{CONFLITO DE INTERESSES}

O autor declara não ter conflitos de interesses.

\section{ENDEREÇO PARA CORRESPONDÊNCIA}

Casimiro Cavaco Dias

UN Building, Major Kanhangulo 197, 3243 Luanda, Angola

Email:diasc@who.int

Recebido em 15-10-2015

Aceite para publicação em 29-06-2016

Artigo redigido ao abrigo do acordo ortográfico anterior a 1990.

\section{ABSTRACT}

\section{THE VALUE CHAIN IN HEALTH: MAKING HEALTH SYSTEMS MORE SUSTAINABLE}

In the current economic crisis, health systems in Portugal and elsewhere in Europe have attempted to increase efficiency in the delivery of health services. However, current approaches to evaluate costs constitute a barrier for creation of value. They lead to cost control measures, which might be ineffective.

Cost control without assessment of health outcomes is counterproductive. Although it might result in potential short-term savings, it may limit effectiveness and compromise long-term sustainability. There is evidence that investment in primary health care and disease prevention can reduce the increasing pressure on emergency services, which are more expensive.

Attention to health outcomes in relation to costs should define the structure for improvements in the health care system. Focusing on the value of health can release the existing potential for innovation in Portuguese society. A more innovative society will be healthier, more productive, and more resilient to future financial crises.

Keywords: Innovation; Medical technologies; Sustainability; Portugal; National Health Service. 